\title{
Surgimiento y creación del concepto de Mesolítico
}

\author{
Mariano Ayarzagüena Sanz ${ }^{1}$
}

\begin{abstract}
RESUMEN
ABSTRACT

En el presente artículo se analiza In this article it is analysed how the cómo surgió el concepto de Mesolítico, las implicaciones ideológicas que la admisión de tal concepto conllevaba en el momento de su creación, asi como el significado que se daba a dicho término y cómo varió a lo largo del siglo $x I X$, según se fueron realizando nuevos descubrimientos.

\section{PALABRAS CLAVE} Mesolítico, Tres Edades, Westropp, Vilanova, Lartet, Mortillet, Siglo XIX. Mesolithic concept appeared, the ideological implications due to the admission of this concept at the moment of its creation, as well as the meaning which this term takes and how it changes along the XIX century as new discoveries were done.

\section{KEYWORDS}

Mesolithic, Three Ages System, Westropp, Vilanova, Lartet, Mortillet, Nineteenth Century.
\end{abstract}

\section{LAS DIVISIONES DE LA EDAD DE PIEDRA ANTES DE 1866}

Las idea de dividir los tiempos prehistóricos en tres edades, de la Piedra, del Bronce y del Hierro fue sugerida por el poeta latino Lucrecio (99 a.C.-55 a.C.), quien definía así lo que ahora denominamos como Edad de Piedra (Lucrecio, 1987: 183): «Las armas primeras fueron las manos,

1 Sociedad Española de Historia de la Arqueología. IES Juan Carlos I. C/ San Francisco s/n. 28350-Ciempozuelos (Madrid). 
las uñas y los dientes, las piedras y también las ramas arrancadas de los árboles, y las llamas y el fuego, desde que fueron conocidos".

No parece que dicha clasificación tuviera mayor repercusión hasta el Siglo de las Luces. Entonces, algunos autores como el francés Antoine Yves Goguet (1716-1758) en 1738 (Daniel, 1981: 55), el danés Shum, o el español Marín y Mendoza ( $i-1776)$, plantearon las tres fases por las que habría pasado el ser humano según Lucrecio, copiando claramente el contenido y redacción del texto de este autor (Marín y Mendoza, 1776: 7). Pero dicho planteamiento lo realizaron desde una óptica exclusivamente teórica, no con la intención de utilizar la clasificación de las tres edades en el análisis de las escasas excavaciones arqueológicas que se llevaban a cabo ya en esos momentos.

Fue Christian Jurgensen Thomsen (1785-1865) quien primero utilizó el Sistema de las Tres Edades con fines prácticos al ordenar los materiales prehistóricos existentes en el Museo Nacional de Antigüedades Danesas de Copenhague, museo que fue abierto al público utilizando esa clasificación en 1819, llegando incluso a exponer en salas diferentes los objetos de cada uno de los periodos, lo que sirvió para divulgar el Sistema de las Tres Edades.

La Edad de Piedra muy pronto empezó a subdividirse. Ya durante la primera mitad del siglo XIX diversos prehistoriadores franceses habían señalado la existencia de dos períodos bien diferenciados en la Edad de Piedra, distinguiendo el terreno "diluvial» o de acarreo antiguo, del "aluvial» o de acarreo moderno, es decir se primaban los criterios estratigráficos, si bien se observaba que en unos niveles aparecían los útiles tallados, mientras que en los modernos se encontraban también útiles pulimentados $^{2}$, por lo que siguiendo otro criterio, el técnico, los prehistoriadores franceses dividian las edades de la piedra en dos: una que llamaban periodo de la piedra tallada y otra en periodo de la piedra pulimentada. Debemos destacar que todavía la Prehistoria no había obtenido un reconocimiento por parte de la comunidad científica, por io que todas estas iniciativas se realizaban al margen, o eran paralelas, a las instituciones oficiales.

Uno de los primeros en establecer esa diferenciación técnica entre los objetos tallados y los pulimentados fue el francés Casimir Picard (18061841) (Richard, 1992: 10), quien integrando datos geológicos, reflexiones

2 Es decir, ya desde antes incluso de obtener su reconocimiento la Prehistoria, se formaron dos tendencias no siempre compatibilizadas, la que recurría a criterios técnicos y aquella que recurría a criterios paleontológicos y geológicos (Groenen, 1994: 155). 
tipológicas y análisis estratigráficos sostenía una cronología muy antigua para los primeros restos humanos (Schnapp, 1993: 292).

A partir de 1859, cuando la Prehistoria alcanzó cierto consenso entre los científicos, primero entre los ingleses y luego ente los franceses, la Prehistoria tuvo un crecimiento vertiginoso. $Y$ se dio nombre a los dos periodos en que vimos se subdividía la Edad de Piedra, Paleolítico y Neolítico. Esta denominación utilizando términos griegos apareció por primera vez en 1865 en la obra de John Lubbock (1834-1913), Prehistoric Times, que alcanzaron un reconocimiento internacional, y hoy en día es como se conocen ${ }^{3}$. El criterio era exclusivamente técnico, y no hacía referencia a la Geología ni a la Paleontología, pues hacía hincapié en la forma de trabajar la piedra, bien mediante la talla; o bien mediante el pulimento.

Como la Prehistoria se originó tomando los métodos y el lenguaje de la Geología y de la Paleontología, por ser esencialmente geólogos, paleontólogos e ingenieros de minas quienes desarrollaron dicha disciplina, también se realizaron otras clasificaciones teniendo en cuenta los criterios geológicos y paleontológicos. Eduardo Lartet (1801-1871) dividió el Paleolítico en cuatro periodos según la fauna existente en el yacimiento: 1) La Edad del Gran Oso de las Cavernas; 2) La Edad del Elefante y del Rinoceronte; 3) La Edad del Reno; y 4) La Edad del Auroch (Lartet, 1861: 217-232). Tras una matización planteada por Garrigou, la clasificación quedó como sigue: 1) Período del Hipopótamo (que sería equivalente al Paleolítico Inferior); 2) Período del Mamut y del Oso de las Cavernas (que sería equivalente al Paleolítico Medio); y 3) Período del Reno (que sería equivalente al Paleolítico Superior).

\section{SURGIMIENTO DEL CONCEPTO DE MESOLITICO EN EL SIGLO XIX}

Hemos expuesto cómo el Sistema de las Tres Edades propuesto por Thomsen muy pronto, antes de 1850, pasó a ser de cuatro, pues la Edad de Piedra pasó a dividirse en dos. Nada más mediar el siglo XIX se presentó un nuevo problema que consistía en ubicar los "kjökkenmöddinger" o "Concheros" en dicha periodización. En 1851 surgió la controversia sobre los "kitchen midden", según la terminología inglesa, que eran enormes concreciones de conchas en la costa de Dinamarca. Mientras,

3 También se utilizó durante varios años la denominación de Arqueolítico como sinónima de Paleolitico, pero al final perdió validez. lgualmente se puede leer en algunas publicaciones del siglo XIX el periodo Eolítico. Este periodo, denominado así por Mortillet, se corresponderia con la supuesta existencia del hombre terciario. 
Japetus Steenstrup (1813-1897) defendía que los "kjökkenmöddinger» eran debidos a la acción natural del oleaje, Jens Worsaae (1821-1885), discípulo de Thomsen, demostró la existencia de un periodo inmediatamente previo al Neolítico, a la que correspondería esa cultura. En el fondo estaba intuyendo la existencia del Mesolítico, si bien, Worsaae nunca llegó a denominar así a este periodo de la Edad de Piedra (Bahn, 1996: 9091). Worsaae utilizó para llegar a esa conclusión los datos procedentes de las excavaciones arqueológicas, elemento nuevo en relación al trabajo de Thomsen, quien se había limitado a clasificar los objetos (Schnapp, 1993: 302).

La primera determinación del Mesolítico data de 1866, es decir tan sólo un año después de que John Lubbock diera a las dos épocas en que se dividía a la Edad de Piedra el nombre de Paleolítico y Neolítico. El nombre de Mesolítico surgió de la pluma del arqueólogo irlandés Hodder Westropp (1820-1885) (Westropp, 1866), y en un principio tuvo muy escaso éxito por las razones que más adelante se verán, de tal forma que ni siquiera quienes usaban el término mencionaban de dónde lo habian tomado, como es el caso de Reboux o de Vilanova.

Westropp era, como la mayoría de los científicos del siglo XIX, un positivista convencido, y planteaba una división de la Edad de Piedra en tres fases, según los distintas formas de fabricar los útiles de ese periodo, y que él entendía se correspondían con tres fases de civilización, visibles en el hombre según los instrumentos utilizados:

1. Artefactos de pedernal de las graveras, evidentemente usados por el hombre en su grado más bajo y más bárbaro de civilización.

2. Artefactos de pedernal encontrados en Irlanda y en Dinamarca, los cuales habian pertenecido a personas que vivian mediante la caza.

3. Artefactos de piedra pulimentada, la cual marca un grado más avanzado, quizás una época pastoril.

Y a cada una de estas fases las denominaba (Westropp, 1866: 291): Paleolítico, Mesolítico y Kainolítico ${ }^{4}$. Ideológicamente, la creación del término intermedio, o Mesolítico, se ajustaba plenamente a su modelo de los Ciclos de Desarrollo (Nicholson, marzo 1983). En este modelo se proponía la unidad psíquica de la especie humana, lo que suponía que todos los pueblos eran, en su conjunto, igualmente inteligentes y capaces de desa-

4 El término Kainolítico fue pronto olvidado, abandono que fue llevado a cabo incluso por su autor (Daniel, Glyn, 1981: 102). 
rrollar los mismos artefactos. En cada ciclo se repetiría el mismo proceso: ascensión, madurez, decadencia, deterioro y transferencia, por lo que no podian existir rupturas.

Lógicamente, con la creación de este Mesolítico, que hoy se correspondería aproximadamente con nuestro Paleolítico Superior, se planteó un debate que se mantuvo prácticamente durante todo el siglo XIX, consistente en especular si habria existido un «hiatus» antes del Neolítico o no. Debemos reflexionar sobre el hecho de que aunque los prehistoriadores del siglo XIX fueran positivistas convencidos en su mayoria, y afirmaban que basaban sus teorías en hechos, sin embargo no existían datos suficientes para poder llegar a conclusiones más o menos consistentes, sirviendo entonces los escasos datos tangibles más a defender las posturas preconcebidas sobre el origen y naturaleza del ser humano que poseían, que para plantear una teoría sólida, lo que entonces resultaba imposible. Asi pues, Westropp, a la par que hacia alarde de sus posturas positivistas no explicaba en su memoria cuáles eran las colecciones o los yacimientos que le habían llevado a realizar tal clasificación.

Uno de los aspectos de fondo más importantes en este tema es el que hace referencia al evolucionismo. Mientras que los evolucionistas defendían la existencia del «hiatus", por cuanto implicaba que a una cultura más desarrollada debía corresponder necesariamente un tipo humano más evolucionado, aquellos que no eran evolucionistas, como era el caso de Juan Vilanova ${ }^{5}$ en España, planteaban que el ser humano habia poseído siempre las mismas potencialidades, por lo que defendian un progreso cultural de carácter más autóctono, muy acorde con el Ciclo de Desarrollo propuesto por Westropp. Decía Vilanova en su curso del Ateneo en 1875 (Vilanova y Piera, 21-1I-1875): “... la especie humana, desde que apareció en el mundo, ostentó sin interrupción todos los rasgos característicos iguales a los que hoy ofrece...".

Por otra parte, Westropp era consciente de la debilidad de las afirmaciones que hacia en su memoria, y no fueron muchos los apoyos que obtuvo dentro de la Sociedad Antropológica de Londres tras su lectura. Así pues, al año siguiente presentó otra comunicación (Westropp, 1867) en la que pretendió demostrar cómo los diferentes instrumentos marcaban una gradación en el estado general de civilización, sin embargo tampoco tuvo mayor éxito entre los miembros de la Sociedad. Si que alcanzaron sus

\footnotetext{
5 Entre los prehistoriadores es muy difícil encontrar fixistas puros. Más bien, como es en el caso de Vilanova, aquellos que no eran evolucionistas convencidos, se inclinaban hacia un eclecticismo, con mayor o menor tendencia, según los autores, hacia el fixismo.
} 
postulados un mayor reconocimiento con otras dos comunicaciones presentadas a la Sociedad Antropológica en $1869{ }^{6}$. En 1872 daba a la imprenta su obra Prehistoric phases, or Introductory Essays in Prehistoric Archaeology, donde proponía su clasificación de una forma mucho más elaborada y donde contaba ya con algún apoyo a sus propuestas, incluso internacional (Nicholson, 1983: 208), pero si leemos las publicaciones de la época podemos observar su escasa repercusión real entre los prehistoriadores, incluso en personas que en cierta forma se encontraban próximos a su mentalidad, como era el caso de John Evans (1823-1908), que establecían una fase intermedia entre el Paleolítico y el Neolítico. Evans ${ }^{7}$, en el Congreso Internacional de Antropología y Arqueología Prehistóricas celebrado en Norwich-Londres en 1868, no utilizaba el término Mesolítico, sino que denominaba a esa fase intermedia Período del Reno o de las Cavernas (Evans, 1869), la cual quedaría bien definida en un área concreta de Europa (Francia Central), y se caracterizaría por el uso de piedras diferentes al guijarro y una mayor habilidad en la talla, si bien aún no se sabría pulimentar.

El término Mesolítico alcanzó una mayor difusión internacional gracias a la comunicación que Reboux ${ }^{8}$ presentó en el Congreso Internacional de Antropología y Arqueologia Prehistóricas que tuvo lugar en Bolonia en 1871 (Reboux, 1873a). Allí Reboux, bajo el título "Investigaciones Paleontológicas en la cuenca de París", planteaba la división en tres fases de la Edad de Piedra: Paleolítico, Mesolítico y Neolítico.

Según Reboux durante el Paleolítico se comenzaría a fragmentar la piedra en láminas y lascas. Durante el Mesolítico los instrumentos se harían en madera, en cuerno, en hueso y en conchas, pero siempre con el auxilio de la piedra, la cual a ella misma se la cambiaría de forma, tallándose toda ella alrededor y tomando la forma del hacha (fig. 1). Diversa fauna, como los elefantes, los ciervos, los caballos, los bueyes y los rinocerontes serían reemplazados por los renos. Tras la mejora de las temperaturas se llegaría al Neolítico, con el cambio de fauna

6 A few words on the origin and development of language y On the mythic age.

7 Evans fue uno de los primeros prehistoriadores ingleses. A principios de los años 60 del siglo $\times 1 \times$ buscó indicios del hombre primitivo en Inglaterra, si bien también dedicó sus esfuerzos a Arqueologia romana. Nombrado caballero en 1892, fue el inicio de una gran saga de arqueólogos, destacando entre ellos su hijo Arthur (Jonh Evans).

8 Reboux fue un naturalista y prehistoriador, miembro de la Sociedad Antropológica de Francia y de la Sociedad Parisina de Arqueología y de Historia. Su principal línea de investigación consistió en el estudio de las relaciones existentes entre los recursos alimenticios durante el Cuaternario y el hombre prehistórico. Merece destacarse para el tema que nos ocupa sus obras Les trois époques de l'âge de pierre (1873) y Chronologie de la pierre (1874). 


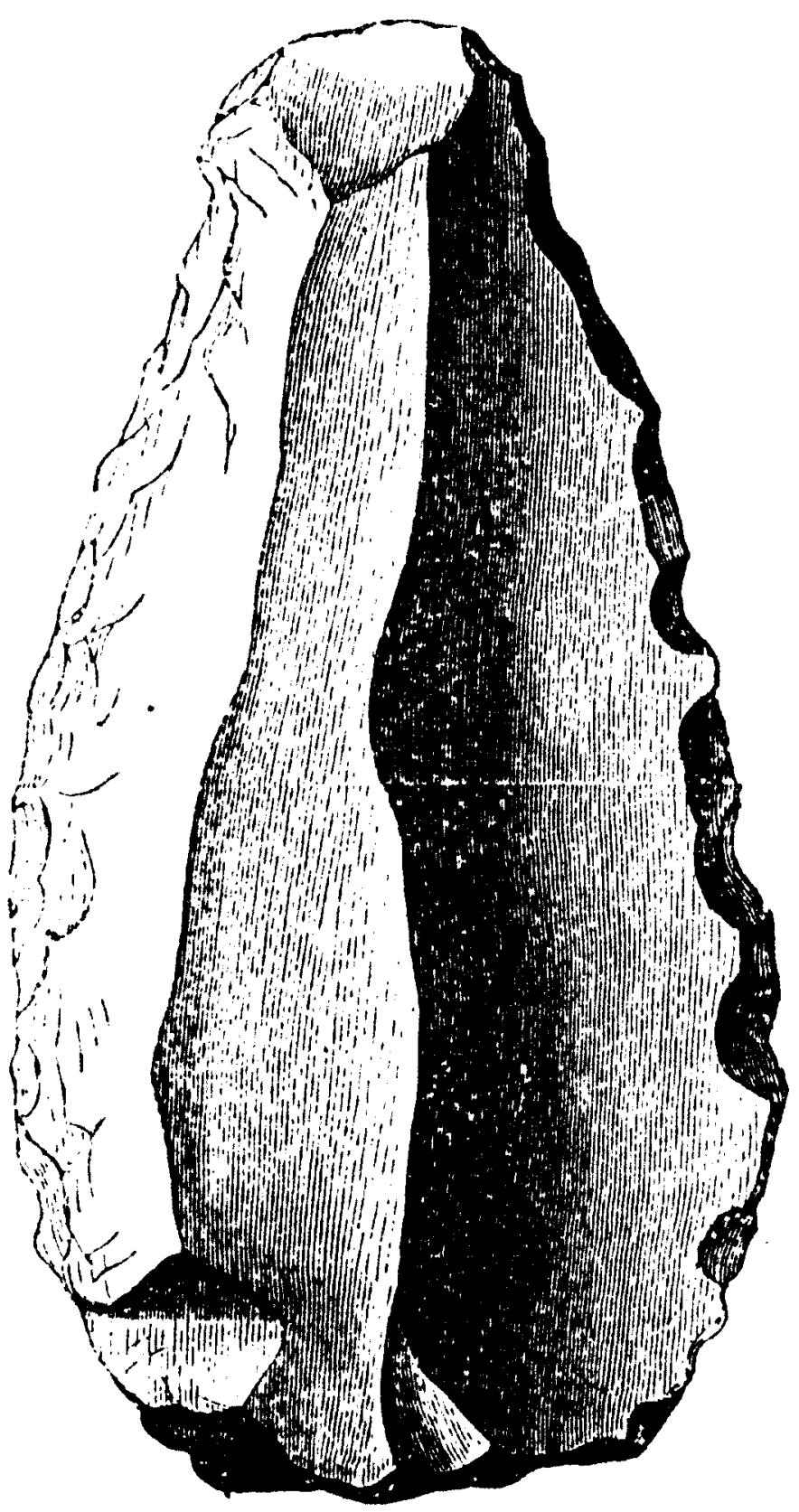

Fig. 1. Epoque Mésolithique 


\section{Cuadro 1. Classification de l'age de la Pierre Par Gabriel de mORTIllet}

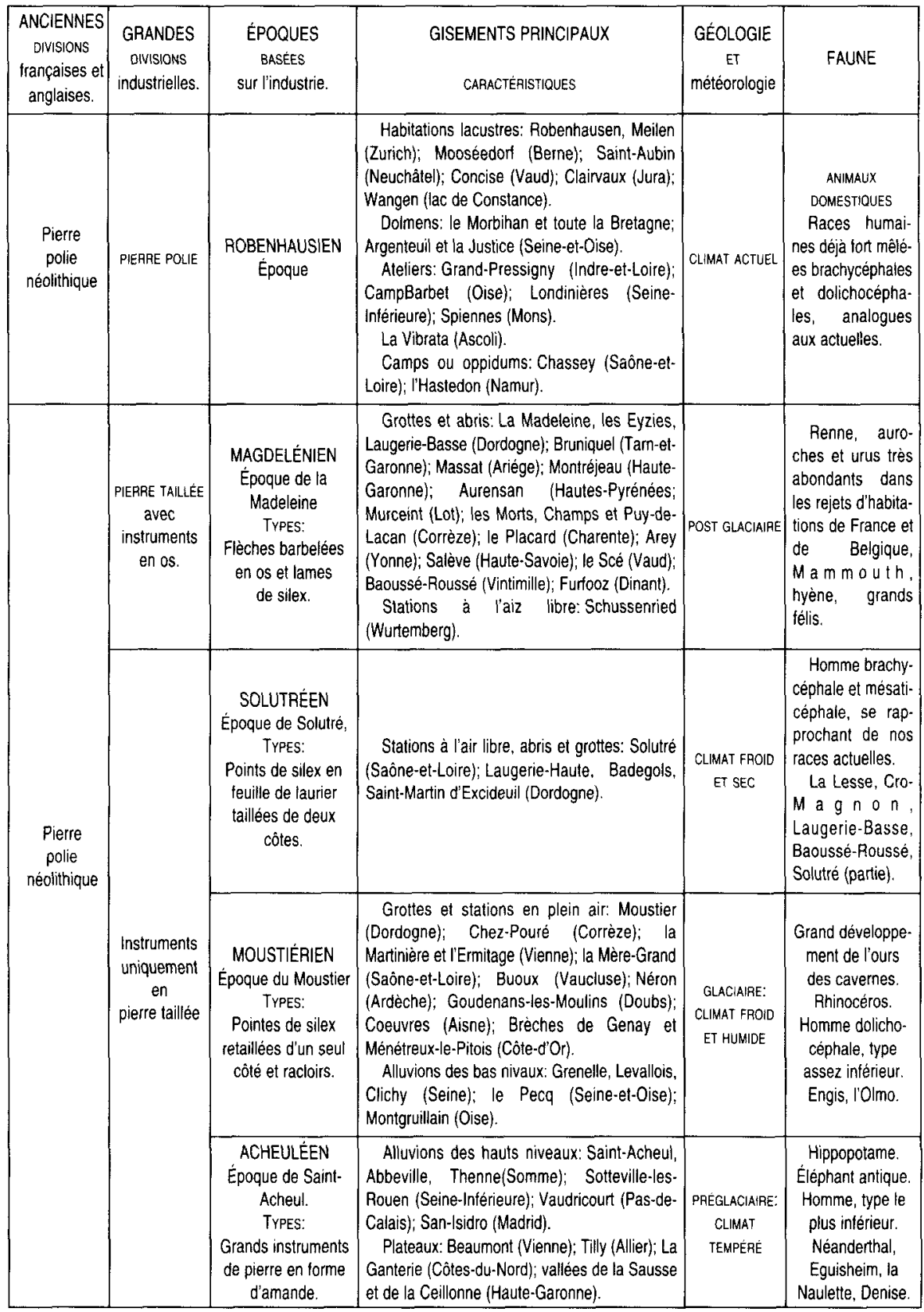


subsiguiente al climático y donde el hombre descubriría el medio de pulir la piedra, utilizando los dólmenes como lugares donde dar sepultura a los antepasados.

\section{SOBRE LA PRETENDIDA EXISTENCIA DE UN HIATUS O LAGUNA}

El debate sobre la existencia, o no, de un periodo intermedio entre el Paleolítico y el Neolítico, o bien sobre la existencia, o no, de un hiatus entre ambos periodos, se plantea directamente en el VI Congreso Internacional de Antropología y Arqueología prehistóricas celebrado en Bruselas en 1872. Aquellos evolucionistas, defensores de la existencia de una laguna entre el Paleolítico y el Neolítico, no dudarán durante el congreso en presentar sus reparos a la existencia del Mesolítico (al que ni siquiera llegan a mencionar), e igualmente, los defensores de la existencia de una continuidad en la cultura y en el ser humano abogarán por rechazar el hiatus mencionado por Gabriel de Mortillet (1821-1898), quien, entre los evolucionistas, destacaba por su prestigio y conocimientos.

Mortillet había realizado en 1867 una clasificación de la Edad de Piedra siguiendo los criterios tipológicos que dio a conocer en su revista Matériaux pour l'histoire positive et philosophique de l'homme (Mortillet, 1867). Pocos años más tarde, en 1869, presentó en la Academia de Ciencias de París una clasificación de la Edad de la Piedra (Mortillet, septiembre de 1872) (cuadro 1), que publica en la misma revista en 1872, es decir el mismo año que se lleva a cabo el VI Congreso Internacional de Antropología y Arqueología prehistóricas en Bruselas, donde volverá a incidir sobre el tema (Mortillet, 1873). En dicho congreso, tan importante que fue en aquellos momentos por la valoración que se hacía sobre la posibilidad de la existencia del hombre terciario en Thenay ${ }^{9}$, se había creado un apartado específico (el V) para tratar la "Clasificación de las Edades de la Piedra". En la ponencia que presentaba Mortillet podemos observar otros muchos aspectos de interés, además de los estrictamente clasificatorios de los que hablaré más tarde:

a) Se observa la disputa existente entre los arqueólogos ingleses y franceses por el liderazgo de los estudios prehistóricos, manifestando, por

9 La posibilidad de la existencia del hombre terciario en Thenay había sido planteada por Bourgeois en el Congreso Internacional de Antropología y Arqueología Prehistóricas de Paris de 1867. 
ejemplo, en relación a las dos edades de la piedra (Mortillet, 1873: 432): "estas dos divisiones, nacidas en Francia, han sido bautizadas por los ingleses (la cursiva es nuestra) con los nombres paleolítico (piedra antigua) y neolítico (piedra nueva)". Esta confrontación se había hecho especialmente visible unos pocos años antes, con los debates sobre la autenticidad de la mandíbula de Moulin Quignon, "encontrada» por los obreros de Boucher de Perthes (1788-1868) en 1863 (Vayson de Pradenne, 1932: 65-104).

b) El intento de separación de los métodos clasificatorios utilizados por los geólogos y paleontólogos, procediendo a utilizar al mismo tiempo criterios industriales. Podríamos ver aquí uno de los primeros intentos por independizar los campos de la Arqueología prehistórica de los de la Geología y la Paleontología (Mortillet, 1873: 433-434), disciplinas de las que hasta entonces había sido subsidiaria, y sin embargo, el mero hecho de plantear unas estaciones tipo es una clara herencia de la Geologia.

c) En el debate posterior a la ponencia se presentaron duras críticas al planteamiento de la existencia de una especie de lo que llamariamos un «fósil-director» para el Achelense, centrado en el hacha de piedra. El abate Bourgeois (1819-1878) ${ }^{10}$ y Augustus Franks ${ }^{11}$ defendieron que en el Achelense eran muchos y muy variados los útiles de ese período, por lo que no se debería restringir la definición del período a un solo modelo de útil.

Mortillet, planteaba una secuencia para el Paleolítico de Achelense, Musteriense, Solutrense y Magdaleniense que, a grandes rasgos ha llegado hasta el siglo xxI. En el fondo, esta clasificación suponía una reinterpretación de la clasificación de Lartet, pero en términos arqueológicos (Daniel, 1981: 64), de tal forma que la Edad del Hipopótamo se convertiría en el Chelense y la Edad del Mamut y del Oso de las Cavernas pasaría a ser el Musteriense. Pero tiene mucho cuidado en deslindar estas divisiones de la existencia de un Mesolítico planteando: "Entre las diversas épocas paleolíticas, se sigue el desarrollo regular y lógico de la industria; se encuentran en ellas transiciones y pasos intermedios. Gradaciones, puntos intermedios, pueden aún faltar, pero se siente, se reconoce que existe una continuidad. No sucede lo mismo entre el

\footnotetext{
10 Gran impulsor que fue de la idea de la existencia del hombre terciario.

11 Conservador del British Museum, era amigo personal de Westropp (Nicholson, 1983: 208).
} 
Paleolítico y el Neolítico, entre el Magdaleniense y el Robenhausiense ${ }^{12}$. Existe alli una gran y profunda laguna, un gran hiatus. Hay una transformación completa". De hecho, al igual que le sucedió a Lubbock, murió sin llegar a reconocer la existencia del Mesolítico, a pesar de que ya en ese momento se había demostrado que con tras el Magdaleniense no se había despoblado Europa, sobre todo tras el descubrimiento de Édouard Piette (1827-1906) en Mas d'Azil en 1889, descubrimiento del que se tratará más adelante.

Tras la lectura de la comunicación, Mortillet sufrirá las críticas de Bourgeois y de Franks, pero no exactamente para tratar el tema de la pretendida laguna, sino para rebatir la existencia de algunas de las fases antes mencionadas. Por el contrario, Mortillet contará con el apoyo de Cartailhac (1845-1921). Un interesante caso de oposición fue el de Paul Broca (1824-1880) (Broca, 1873), por cuanto nos indica el estado aún incipiente en el que se encontraba la ciencia prehistórica en ese momento. Broca mantenía la continuidad desde el Paleolítico hasta el Neolítico, utilizando para ello el yacimiento de Aurignac ${ }^{13}$, exponiendo que en dicho yacimiento se entremezclaban los útiles tallados con los pulimentados y los enterramientos se habian realizado en cueva y no en dólmenes, como se entendía que de forma exclusiva se enterraba durante el Neolítico. Con esos datos ubicaba a esa cultura a medio camino entre el Paleolítico y el Neolítico. De todas formas debemos tener en cuenta que la correcta clasificación del Auriñaciense no se realizó hasta principios del siglo xx, gracias a las investigaciones de Henri Breuil (1877-1961).

En la misma línea, Edouard Dupont (1841-1911), defendiendo el principio de las evoluciones técnicas paralelas (Dupont, 1873), consideraba al «hombre de la piedra pulida como el descendiente directo, en las mismas regiones, de las poblaciones de los aluviones cuaternarios de los valles del Sena, del Somme o del Haine» (Dupont, 1873: 478).

12 Se denominaba Robenhausiense a una cultura que entonces se clasificaba como perteneciente al Neolítico. Se describió a partir del yacimiento epónimo de Robenhausen, aldea perteneciente a Wetzikon, en el cantón de Zúrich (Suiza) y que habia sido explorada y descrita principalmente por Jacob Messikommer y Ferdinand Keller (1800-1881), tras los descubrimientos realizados en ese lugar desde 1857, si bien tanto Messikommer como Keller ya llevaban trabajando en los yacimientos palafíticos suizos desde hacia años.

13 El yacimiento de Aurignac lo había empezado a excavar Eduardo Lartet en octubre de 1860, pero cuando él llegó a ver los enterramientos que en él se contenían, a los huesos se les había dado ya cristiana sepultura, por lo que realizó excavaciones en la cueva y preguntó a los operarios que encontraron los restos humanos por aquellos datos que le podían resultar de interés, pero los obreros no habian retenido en la memoria todos los datos que se les preguntaba (Lartet, 1861: 179). 
Pero el único que en dicho congreso volvió a aludir al Mesolítico de forma extremadamente escueta, pero explícita, fue Reboux (Reboux, 1873b), quien definió tipológicamente de una forma más confusa cada uno de los periodos, pero los interrelacionó mucho más con la fauna. Así al Paleolítico lo relacionaba con el Mamut y el Oso de las Cavernas y tipológicamente con las lascas de piedra. Al Mesolítico con el reno y la piedra taliada y al Neolítico con los dólmenes y la piedra pulimentada.

Con las posturas tan encontradas la situación quedó en tablas. Es cierto que el prestigio de Mortillet era tal que rara vez se volvió a utilizar el término de Mesolítico en los siguientes años hasta finales de los años 80 , pero no por ello se dejó de plantear la continuidad desde el Paleolítico hasta los tiempos actuales por parte de algunos arqueólogos europeos, entre los que podemos contar a los más importantes españoles, como se verá a continuación. De todas formas, en el Congreso de Estocolmo de 1874 volvió a ser usado el término por Otto Torell, profesor de Geología en Lund (Daniel, 1981: 102).

Por último, otra de las líneas de investigación abiertas desde la que se especulaba con la existencia de un paso directo desde el Paleolítico al Neolítico, fue la propuesta por el antropólogo americano Lewis $\mathrm{H}$. Morgan (1818-1881). Este antropólogo publicó en 1877 una obra, Ancient Society (Morgan, 1877), que provocó una fuerte polémica al plantear que los sistemas humanos de emparejamiento habian evolucionado pasando por unas etapas concretas y sucesivas de promiscuidad, matrimonio en grupo, poligamia y monogamia. En esta obra planteaba que el Sistema de las Tres Edades fundamentado en caracteres técnicos había sido muy útil para ciertos propósitos, y estimaba que se seguiría usando para aspectos tales como la clasificación de objetos de arte antiguo, pero que para el estudio de las sociedades sería mucho más útil realizar otro tipo de subdivisiones. Morgan planteaba la existencia de siete periodos:

1. Nivel más bajo de Salvajismo, que abarcaría desde la infancia de la raza humana hasta la adquisición de un pescado como subsistencia y del uso del fuego.

II. Nivel Medio de Salvajismo, que finalizaría con la invención del arco y la flecha.

III. Nivel Superior de Salvajismo, que llegaría hasta la invención del arte de la cerámica.

IV. Nivel inferior de Barbarie, que abarcaría en Europa hasta la domesticación de animales y en América hasta el cultivo del maíz y de las plantas por irrigación. 
V. Nivel Medio de Barbarismo, que llegaría hasta la fundición del hierro.

VI. Nivel Superior de Barbarismo, que finalizaría con el uso de la escritura con composición literaria, y

VII. Nivel de Civilización, que sería desde que el ser humano conociera la escritura como composición literaria en adelante.

Es de señalar, que todos los periodos se inician donde ha acabado el anterior, lo que viene a mostrar una linealidad evolutiva y un fuerte autoctonismo. El Mesolítico se correspondería con el nivel superior del Salvajismo, y en el texto se muestra los debates que se están realizando en ese momento desde el campo de la Prehistoria sobre el pretendido «hiatus". Morgan al hablar del nivel más bajo de Barbarie (nivel IV), dice que el periodo comienza con la manufactura de la cerámica, tanto «si es por invención original o por adopción” (Morgan, 1877: 10). Esta diferencia entre invención original o adopción en uno de los periodos, Morgan lo plantea en su clasificación sólo en este periodo.

\section{LA LLEGADA DEL CONCEPTO DEL PERIOODO MESOLITIICO A ESPAÑA}

En España, existía desde principios de los años 60 del siglo xIX la idea de que el ser humano había pervivido en la Península lbérica desde los más remotos tiempos hasta la actualidad. Casiano de Prado (1797-1866), por ejemplo, planteaba la existencia de una Edad del Cobre previa a la Edad del Bronce (Prado, 1864: 200) y que se situaría ente el Neolítico y la Edad del Bronce. Igualmente en España se dio muy buena acogida al término de Mesolítico por parte de Juan Vilanova desde $1872 \mathrm{y}$, de hecho, este autor nunca lo olvidaría utilizándolo siempre en sus clasificaciones.

En el ya citado año de 1872, Vilanova publicó su obra Origen, naturaleza y antigüedad del hombre, asi como diversos artículos en revistas como en los Anales de la Sociedad Española de Historia Natural o el Museo Español de Antigüedades, en los que prácticamente repite el mismo texto en todos ellos. Divide la Edad de Piedra en Paleolítico y Arqueolítico; Mesolítico y Neolítico. Podemos observar mejor la clasificación de Vilanova, en el cuadro 2.

En sus obras de 1872, al iniciar el tratamiento del Mesolítico dice “... la mayor parte de los documentos prehistóricos hasta el presente encontrados en la Península, pertenecen de lleno a ese periodo y al inmediato, siendo sus principales yacimientos la cueva, los dólmenes y ciertos depósitos ex- 


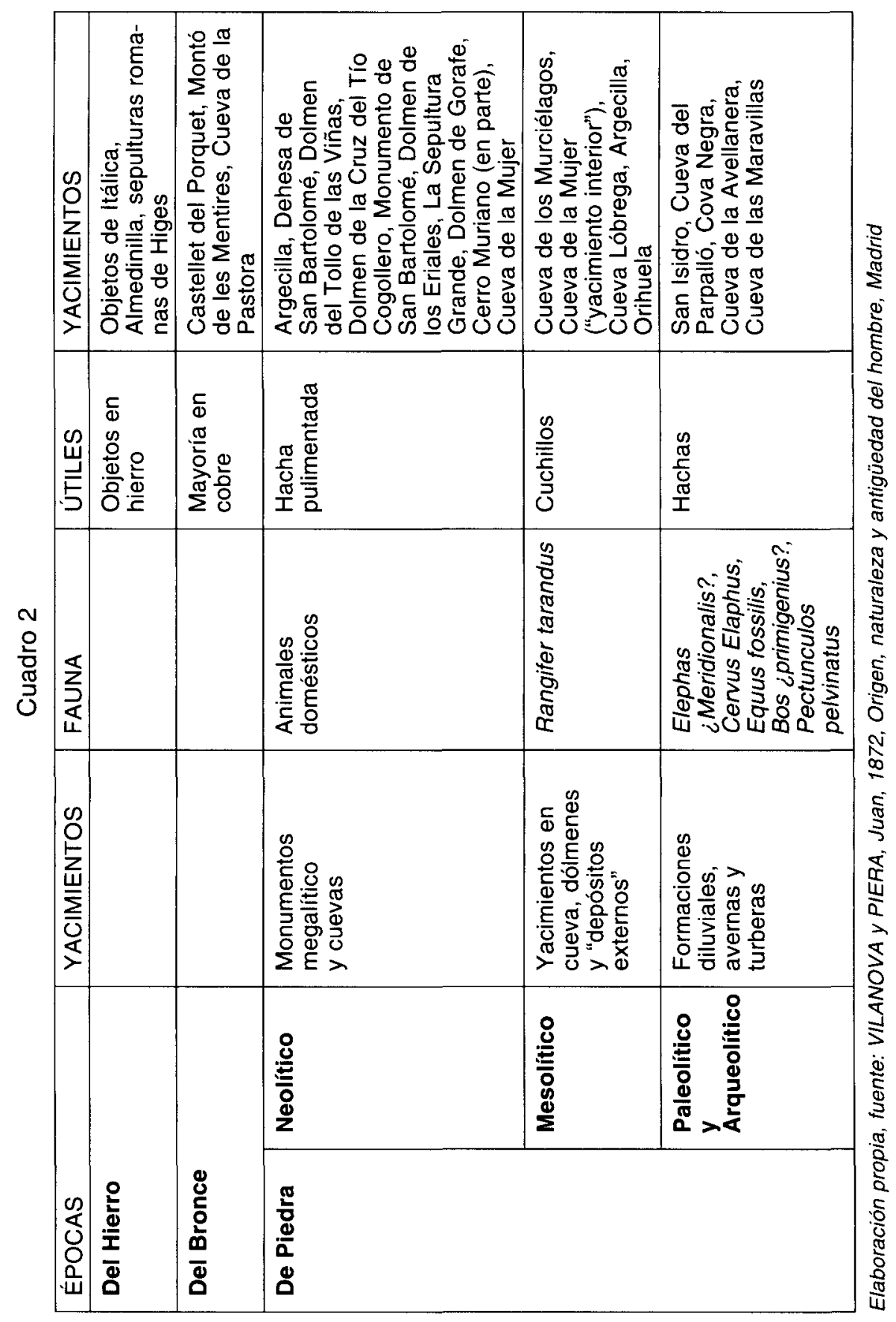


ternos análogos a los encontrados en Italia y Suiza, ...». Es decir, como ya dije anteriormente, ante la situación de infancia en que se encontraba la Prehistoria, los datos eran muy escasos y era factible, como en este caso, que se interpretaran mal según los intereses ideológicos de quien los analizaba y así, como en este caso, surgía un periodo intermedio entre el Paleolítico y el Neolítico artificialmente, al mezclar objetos pertenecientes a ambos periodos. Así pues, si bien es cierto que estaba muy acertado en cuanto a la existencia del Mesolítico, lo confundía con parte de lo que hoy denominamos Paleolítico Superior y mezclaba su industria con otra neolíti$\mathrm{ca}$, e incluso posterior (fig. 2 y 3$)^{14}$. Por consiguiente, obviaba todo el periodo realmente mesolítico, como quedaría claro unos pocos años más tarde tras las excavaciones realizadas por Piette en Mas d'Azil.

Sin embargo, un científico ecléctico, como Vilanova, no desaprovechaba todo aquello que consideraba de interés de otros científicos con los que no compartía su ideología, e incluso lo adaptaba a sus planteamientos teóricos. Tras el Congreso de Estocolmo en 1874, adaptaría la clasificación de Mortillet en las conferencias que impartía en el Ateneo de Madrid, denominando Matritense al Achelense (por San Isidro), Camerense al Musteriense (por Cueva Lóbrega en la Sierra de Cameros), del Solutrense no encontraría correspondencia en España, Argecillense al Magdaleniense (por la cueva de Argecilla en Guadalajara) y Granatiense al Robenhausiense (por la Cueva de la Mujer, en Granada) ${ }^{15}$, y más tarde se referirá al Olleriense (de Olleria, en Valencia) como período de la Edad del Cobre. Si nos fijamos, lo que hace es establecer una nomenclatura local a las épocas propuestas por Mortillet, planteando asi la existencia de una especificidad y un localismo para las industrias de la península lbérica. Pocos años más tarde, en 1883, variaria ligeramente la ubicación cronológica del Mesolítico, entendiendo que se correspondia mejor con el Solutrense, pues su hojas con retoque plano entendía que marcarian una línea que conduciría hasta el Neolítico (cuadro 3). Para Vilanova sería durante el Mesolítico, es decir, durante el Solutrense, cuando se iniciaría la fabricación de vasijas de barro, lo que nos indica el escaso conocimiento que tenía de esta cultura, ignorancia y vacilaciones en este aspecto que mantendria durante toda su vida.

\footnotetext{
14 El yacimiento de Argecilla tiene todo el aspecto de corresponder realmente a la Edad del Bronce y a pesar de este error posiblemente sea el último yacimiento en que Vilanova practicó excavaciones arqueológicas.

15 Información contenida en diversos artículos publicados en Revista Europea III y $\mathrm{IV}$ durante los años 1874 y 1875 bajo el título genérico de "Ciencia prehistórica. Lecciones dadas en el Ateneo".
} 


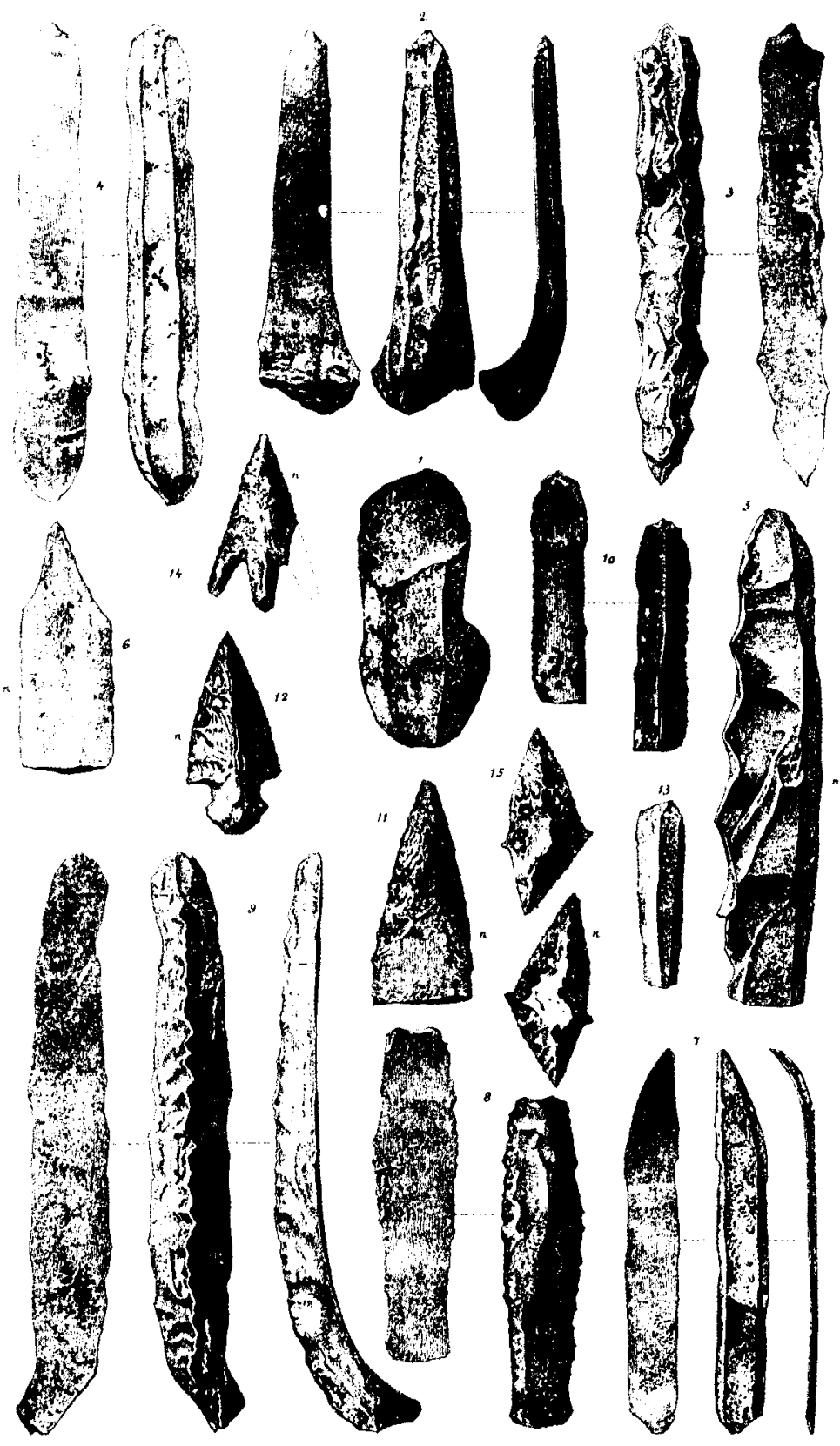

Fig. 2. Cuchillos y flechas de España. Época Mesolítica (sic). 


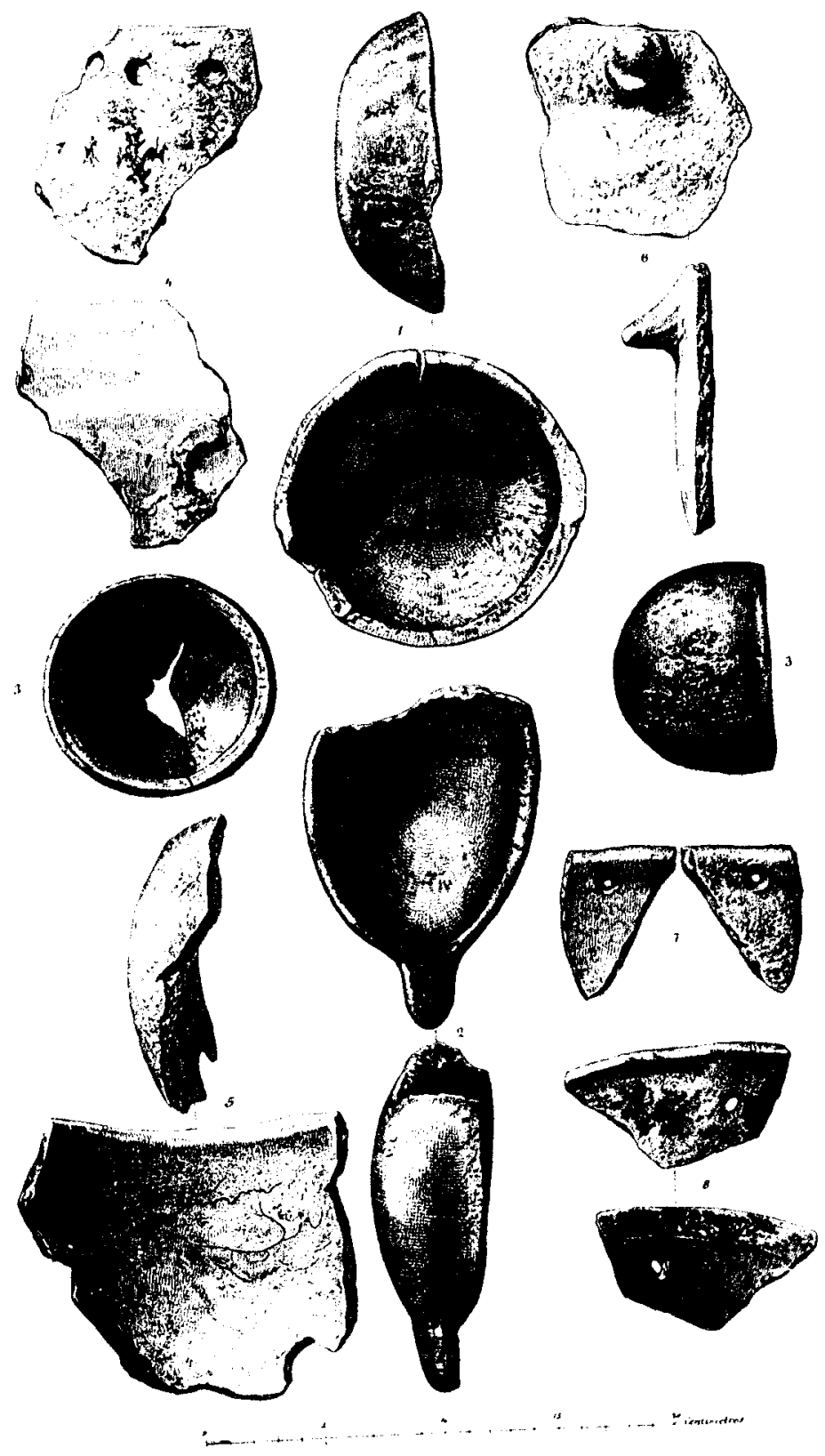

Fig. 3. Cerámica de la Época Mesolitica (sic). 


\begin{tabular}{|c|c|c|c|c|}
\hline 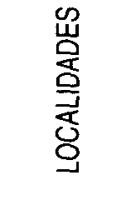 & 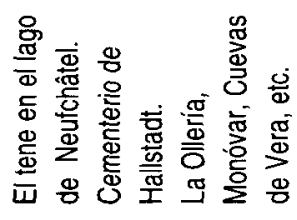 & 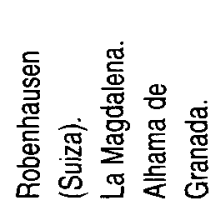 & 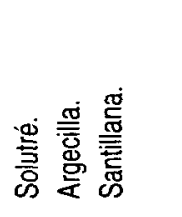 & 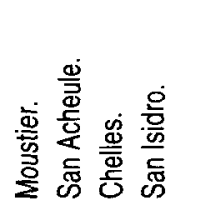 \\
\hline 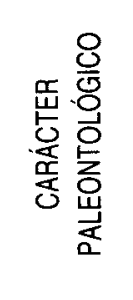 & 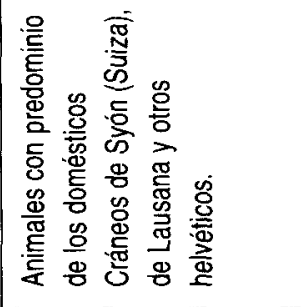 & 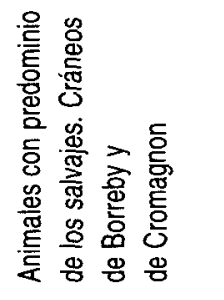 & 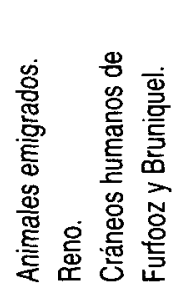 & 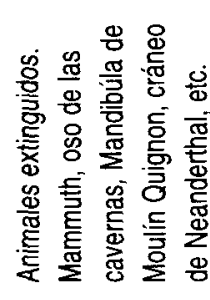 \\
\hline 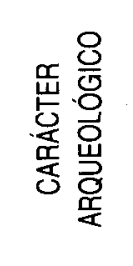 & 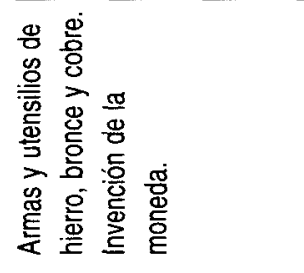 & 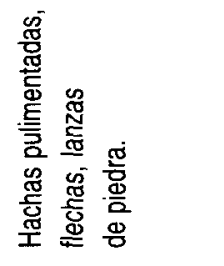 & 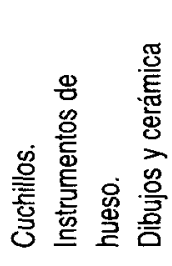 & 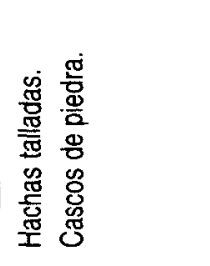 \\
\hline 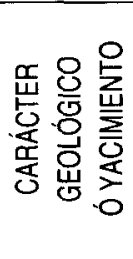 & 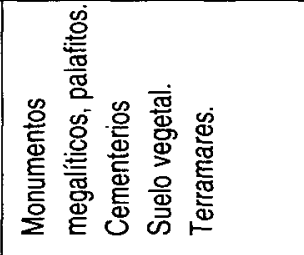 & 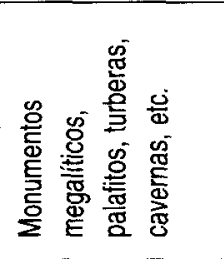 & 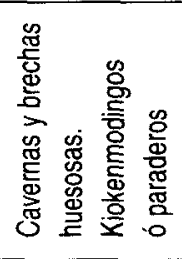 & 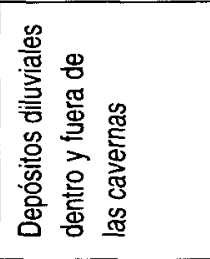 \\
\hline$\stackrel{ }{\stackrel{2}{1}}$ & 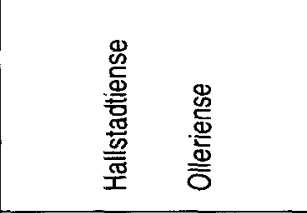 & 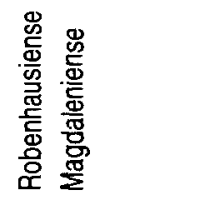 & 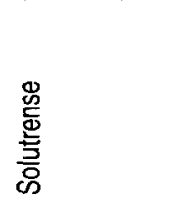 & 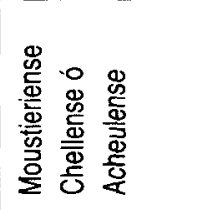 \\
\hline $\begin{array}{l}\mathscr{0} \\
8 \\
\frac{0}{\pi} \\
\text { 폼 }\end{array}$ & 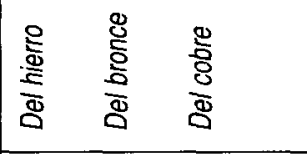 & 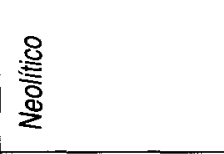 & 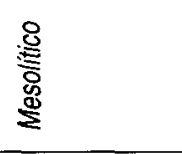 & 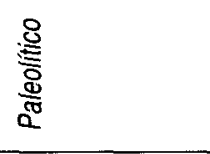 \\
\hline $\begin{array}{l}\text { 岩 } \\
\text { 岂 }\end{array}$ & 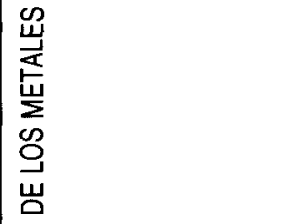 & $\begin{array}{l}\text { 営 } \\
\text { 嵩 } \\
\text { s } \\
\text { 岁 }\end{array}$ & & \\
\hline
\end{tabular}


Y Vilanova no fue el único en España en defender el Mesolítico. También José Joaquín Landerer (1841-1922), en su «Explicación al cuadro sinóptico de los tiempos primitivos" publicada en 1873 (Landerer, 1873), habla de la uedad del Reno ... denominada época de los cuchillos, y por otro nombre mesolítica, por ser intermedia ente la de la piedra tallada primitiva y la de la pulimentada o neolítica, que se enlaza con el periodo histórico por tránsitos insensibles" (Landerer, 1873: 362).

\section{EVOLUCIÓN DEL CONCEPTO DE MESOLITICO DURANTE EL SIGLO XIX}

La existencia de un hiatus entre el Paleolítico y el Neolítico se fue imponiendo y muy pocas voces discordantes, como fue el caso de Vilanova del que ya hemos hablado, permanecieron claramente en la defensa del Mesolítico. Así las cosas, Gabriel de Mortillet (Mortillet, 1883) planteó en 1883 de una manera aparentemente concluyente la existencia de grandes migraciones desde Europa al final de Paleolítico y que en el Neolítico, una raza nueva, procedente de Oriente habria llegado a Europa con una cultura totalmente distinta..

Sin embargo, en 1887 Édouard Piette (1827-1906) descubre una industria postmagdaleniense con característicos arpones, que rápidamente la coloca entre el Magdaleniense y el Neolítico (Piette, 1889a), en Mas d'Azil. Si los estudios sobre el arte paleolítico de este prehistoriador marcaron todo un hito en los estudios prehistóricos, fueron sus «hallazgos en Mas d'Azil los que aportaron a Piette la información más abundante, más original y más determinante en lo que concierne a su pensamiento cientifico" (Delporte, 1987). Piette colocaba a la cultura por él encontrada en un periodo intermedio entre la Edad del Reno (Magdaleniense) y el Neolítico, y no, como había sucedido hasta la fecha, haciéndolo equivalente al Magdaleniense, e incluso al Solutrense. Una vez presentado su hallazgo en la Academia de Ciencias, defiende su hipótesis de la existencia de un periodo de transición en el Congreso Internacional de Antropología de París (Piette, 1889b) y ya de una forma más elaborada en el Boletín de la Sociedad Antropológica de París (Piette, 1895). Por cierto, en otra publicación presentaba los cantos pintados azilienses (Piette, 1896), que en aquel momento era el único tipo de pintura de época anterior al Neolítico que podía ser aceptada, dada la ideología imperante al respecto.

Y no fue este el único yacimiento del Mesolítico que se excavó en los años 80. Philippe Salmon (1823-1900) iniciaba en 1886 excavaciones en 
Campigny donde descubría una industria más reciente que la Magdaleniense, pero anterior al Neolítico que también daba a conocer al final de la década (Salmon, Aul du Mesnil, \& Capitan, 1898). Sin embargo ni Piette ni Salmon utilizaron en un primer momento el término de Mesolítico.

Con todos estos descubrimientos, y la aceptación de culturas como la Aziliense en la cronología prehistórica se asumía una evolución técnica lineal con un aparente frenazo a la evolución, por la apariencia de ser una industria más atrasada, razón por la que Mortillet y Cartailhac (Cartailhac, 1896) seguían mostrándose reticentes hacia el Mesolítico y Lubbock se negó a admitirlo (Rivière, 1978: xvii). Mortillet, poco antes de su muerte propuso un periodo el "Tourassien» al no querer aceptar el término de Aziliense, pues temía que los cantos pintados fuesen una falsificación, por lo que sugería tomar el nombre no de un yacimiento como en él era habitual, sino de una zona, la Tourasse, situada en el Aito Garona (Mortillet, 1910: 321). Este "Tourassien" vendría a cubrir el antiguo hiatus, si bien se seguirían negando los Mortillet, padre e hijo, a incluir el Mesolítico en sus clasificaciones (Mortillet, 1910: 21).

El regreso internacional a la nomenclatura de Mesolítico, parece que se debe al arqueólogo inglés J. Allen Brown en 1892 (Oakley, 1968: 149), sobre todo gracias a los hallazgos antes citados en Francia y a los que el mismo Brown estaba realizando en el valle del Támesis, que le permitieron volver a una nomenclatura que algunos, como Vilanova, nunca olvidaron. Y será ya a principios del siglo xx, con el arqueólogo francés Jacques de Morgan (1857-1924), cuando alcance definitivamente su aceptación internacional (Morgan, 1909).

Las nuevas generaciones de prehistoriadores que surgirán a principios del siglo xx, establecerán definitivamente el concepto de Mesolítico, diferenciándolo del Epipaleolítico. Asimismo, se destruirá progresivamente la cronología de Mortillet, pues si bien es cierto, como se dijo al principio del presente artículo, que las épocas que él inventó conservaron su denominación, sin embargo los principios teóricos sobre las que se construyeron habian desaparecido (Richard, 1992: 33) ya a principios del siglo $x x$, especialmente en todo lo relativo al modelo evolutivo, pues la estricta correspondencia entre una cultura industrial y una época cronológica perdía su sentido al observarse localismos en las distintas partes de Europa, e incluso en la misma Francia; y por otra parte, el darwinismo decimonónico estaba tocando a su fin, e iba a surgir el neodarwinismo desde unos planteamientos ligeramente diferentes, donde se daba cabida al azar y se perdía el determinismo estricto que habia marcado al siglo XIX. 


\section{BIBLIOGRAFÍA}

BAHN, Paul G. (1996): History of Archaeology, Cambridge University Press.

Broca. Paul (1873): "Sur la caverne de l'homme mort près Saint Pierre les Tripiès (Lozère)", Congrés International d'Anthropologie et d'Archéologie Préhistoriques, $6^{\text {ème }}$ session, (Bruselas, 1872), pp. 182-198. París.

CARTAllHAC, Emile de (1896): La France préhistorique d'après les sepultures et les monuments, Félix Alcan, Éditeur, 2. ${ }^{a}$ édition, París.

DANIEL, Glyn (1981): A Short History of Archaeology, Thames and Hudson, Londres.

DelPORTE, Henri (1987): "Piette, pionnier de la Préhistorie", en Edouard PIETTE, Histoire de l'art primitif, Picard, Paris.

DUPONT, Édouard (1873): "Classement des âges de la pierre en Belgique", Congrés International d'Anthropologie et d'Archéologie Préhistoriques, $6^{\text {éme }}$ session, (Bruselas, 1872), pp. 459-479. Paris.

EVANS, John (1869): "On the manufacture of stone implements in prehistoric times", International Congress of Anthropology and Prehistoric Archaeology (Norwich-London, 1868), pp. 191-193, Londres.

GRoEnen, Marc (1994): Pour une histoire de la préhistoire. Le Paléolithique, Éditions Jérôme Millon, Grenoble.

LANDERER, José J. (1873): "Explicación al cuadro sinóptico de los tiempos primitivos", Anales de la Sociedad Española de Historia Natural, Actas, 11, pp. 341-378, Madrid.

LARTET, Edouard (1861): «Nouvelles recherches sur la coexistence de l'homme fossile et des grands mammifères fósiles reputes caractéristiques de la derniére période Géologique", Annales des Sciences Naturelles II, Zoologie, $4^{\mathrm{em}}$ série, XV pp. 177-253.

LuCRECIO (1987): De la Naturaleza, Introducción de José Ignacio CiRUelo Borge, traducción y notas de Eduard VALENTI FIOL. Ed. Planeta, Barcelona.

Marin y Mendoza (1776): Historia de la Milicia española, Madrid.

MORgan, J. de (1909): Les premières civilisations, E. Leroux, París.

Morgan, Lewis H. (1877): Ancient Society or Researches in the Lines of Human Progress from Savagery through Barbarism to Civilization. Charles H. Kerr \& Company, Chicago.

MORTILlet, G. de (1867): «Promenades préhistoriques à l'exposition universelle», Matériaux pour l'histoire positive et philosophique de l'homme, año VII, $2 .^{a}$ serie, t. III, pp. 181-281 y 285-368. París.

Mortillet, G. de, septiembre de (1872): “Classificattion de l'âge de la pierre", Matériaux pour l'histoire positive et philosophique de l'homme, año VII, 2. ${ }^{a}$ serie, t. III, París.

Mortillet, G. de (1873): "Classificattion des diverses périodes de l'âge de la pierre", Congrés International d'Anthropologie et d'Archéologie Préhistoriques, $6^{\text {'me }}$ session, (Bruselas, 1872), pp. 432-444. París.

Mortillet, G. de (1883): Le préhistorique, Reinwald, París.

Mortillet, Gabriel y Adrien de (1910): La préhistoire, Librairie Schleicher Frères, París.

Nicholson, Paul T., marzo (1983): "Hodder Westropp: nineteenth-century archaeologist", AntiquityLVII, n. ${ }^{\circ} 219$, pp. 205-210.

OAKLEY, Kenneth (1968): Cronologia del hombre fósil, Biblioteca Universitaria Labor, Barcelona.

PIETTE, Édouard (1889a): «Un groupe d'assises représentant l'époque de la transition entre les temps quaternaires et les temps modernes", Comptes rendus des séances de l'Académie des Sciences CVIII, p. 422, séance du 25 février, París.

- (1889b), "L'époque de transition entre l'âge du renne et celui de la pierre polie". Congrès International Anthropologique de Paris, pp. 203-209 y 210-211.

- (1895): "Hiatus et lacune. Vestiges de la période de transition dans la grotte du Masd'Azil», Bulletin de la Société d'Anthropologie de Paris, 4éme série, VI, pp. 235-267.

- (1896): «Études d'ethnographie préhistorique. Les plantes cultivées du Mas-d'Azil. Les galets coloriés du Mas-d'Azil», L'Anthropologie VII, pp. 1-17 y 385-427.

Prado, Casiano de (1864): Descripción física y geológica de la provincia de Madrid, Imprenta Nacional, Madrid.

REBOUX (1873a): «Recherches paléontologiques sur le bassin de Paris», Congrés International d'Anthropologie et d'Archéologie Préhistoriques, $5^{\text {eme }}$ session, (Bologna, 1871), pp. 98-102. Bolonia. 
- (1873b): "Collection de silex recueillis dans les carrières de Levallois", Congrés International d'Anthropologie et d'Archéologie Préhistoriques, $6^{\text {eme }}$ session, (Bruselas, 1872), p. 479. París.

RICHARD, Natalie (1992): L'invention de la préhistoire, Presses Pocket, s.l.

RIVIĖRE, Peter (1978): "Introduction", en John LUBBOCK, The Origin of Civilisation and the Primitive Condition of Man, University of Chicago Press.

Salmon, J.; Ault du MEsnil, G. \& CAPITAN, J. (1898): "Le campinien, fouille d'un fond de cabane au Campigny, commune de Blangy-sur-Bresle (Seine-Inférieure)", Revue de l'École d'anthropologie VIII, pp. 365-408, París.

SchnapP, Alain (1993): La conquête du passé. Aux origines de l'archéologie. Editions Carré. París.

VAYSON DE PRADENNE, A. (1932): Les fraudes en archéologie préhistorique, Émile Nourry, Éditeur, Paris.

VILANOVA y PIERA, Juan, 21-II-1875: “Naturaleza y origen del hombre", Revista Europea, $n .^{\circ}$ 52, pp. 550-552, 7. ${ }^{a}$ lección dada en el Ateneo de Madrid el 16-II-1875, Madrid.

WESTROPP, Hodder (1866): «Analogous forms of implements among early and primitive races", Memoirs of the Anthropological Society ii, pp. 288-293.

WESTROPP, Hodder (1867): "On the sequences of the phases of civilisation and contemporaneus implements", Antropological Review $\mathrm{V}$. 